\title{
Theta oscillations predict the detrimental effects of memory retrieval
}

\author{
Simon Hanslmayr, Tobias Staudigl, Alp Aslan, ANd Karl-Heinz BäUml \\ Regensburg University, Regensburg, Germany
}

\begin{abstract}
Retrieving a target item from episodic memory typically enhances later memory for the retrieved item but causes forgetting of competing irrelevant memories. This finding is termed retrieval-induced forgetting (RIF) and is assumed to be the consequence of an inhibitory mechanism resolving retrieval competition. In the present study, we examined brain oscillatory processes related to RIF, as induced by competitive memory retrieval. Contrasting a competitive with a noncompetitive retrieval condition, we found a stronger increase in early evoked theta $(4-7 \mathrm{~Hz})$ activity, which specifically predicted RIF, but not retrieval-induced enhancement. Within the cognitive framework of RIF, these findings suggest that theta oscillations reflect arising interference and its resolution during competitive retrieval in episodic memory. Supplemental materials for this article may be downloaded from http://cabn.psychonomic -journals.org/content/supplemental.
\end{abstract}

Retrieval from episodic memory is constantly challenged by interference from currently irrelevant, related memory traces (Anderson \& Neely, 1996; Bäuml, 2008). For example, if we want to recall what we had for dinner last week at our favorite Italian restaurant, memories related to other dinners in this restaurant interfere. To overcome such interference, the irrelevant memories are supposed to be inhibited, inducing later forgetting of the interfering information (e.g., Anderson, 2003). The aim of the present study was to identify the brain oscillatory correlates of such retrieval-induced forgetting (RIF).

In the laboratory, RIF can be investigated using the retrieval practice paradigm (Anderson, Bjork, \& Bjork, 1994; Figure 1A). In this paradigm, subjects study items from different semantic categories (e.g., FRUIT-orange, FRUIT-banana, INSECT-ant) and subsequently repeatedly retrieve half of the items from half of the categories, using a word stem completion task (e.g., FRUIT-ban__). On a later memory test, the subjects are asked to recall all previously studied items. Relative to the control items from the unpracticed categories (ant), retrieval practice typically improves recall of the practiced material (banana) but impairs recall of the unpracticed material (orange), which is referred to as the RIF finding (for reviews, see Anderson, 2003; Bäuml, Pastötter, \& Hanslmayr, 2010).

It is widely assumed that RIF is caused by inhibitory processes. The proposal is that during retrieval practice on a subset of the studied material, related unpracticed items interfere. To reduce the interference, the unpracticed material is inhibited by deactivation of the unpracticed items' memory representations (Anderson \& Spellman, 1995). Consistently, RIF has been found across a wide range of memory tests, including cued recall tests (e.g., Anderson et al., 1994), independent-probe tests (cues not presented in a prior phase of the experiment; e.g., Anderson \& Spellman, 1995), recognition tests (e.g., Spitzer \& Bäuml, 2007), and implicit tests (e.g., Veling \& van Knippenberg, 2004). In particular, RIF is a recall-specific effect. Accordingly, inhibitory effects of practice in episodic memory have been observed in retrieval practice conditions, but not when a subset of the material was reexposed or learned on a second instance (Bäuml \& Aslan, 2004; Ciranni \& Shimamura, 1999; for related results, see Anderson, Bjork, \& Bjork, 2000; Bäuml, 2002).

Investigating the neural mechanisms mediating RIF, two recent studies compared neural activity in a retrieval practice condition with neural activity in a reexposure condition (Johansson, Aslan, Bäuml, Gäbel, \& Mecklinger, 2007; Wimber, Rutschmann, Greenlee, \& Bäuml, 2009). Using electrophysiological measures of brain activity, Johansson et al. observed increased sustained positivity over frontal electrode sites during the retrieval practice phase, which predicted later forgetting. Using fMRI, Wimber et al. (2009) showed that retrieval practice leads to a BOLD signal increase in left prefrontal and parietal brain regions. Thereby, prefrontal activations in the dorsolateral prefrontal and anterior cingulate cortex (ACC) predicted later forgetting. Examining the effects of repeated retrieval practice, Kuhl, Dudukovic, Kahn, and Wagner (2007) demonstrated that the BOLD signal in lateral prefrontal brain regions and the ACC decreased with an increasing number of retrieval attempts, which again correlated with later forgetting. Together, these studies point to a major role of the ACC and the lateral prefrontal 
A

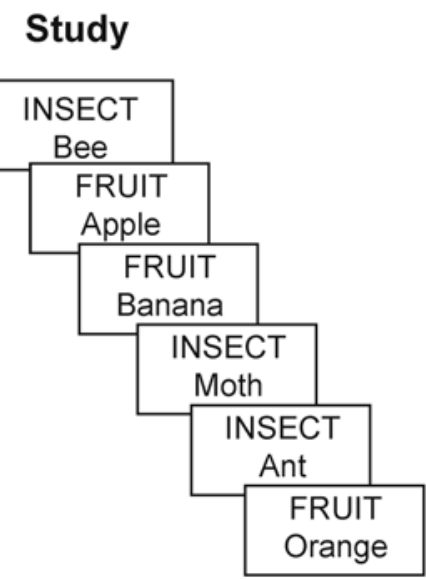

\section{EEG Recording}

\section{Retrieval Practice}

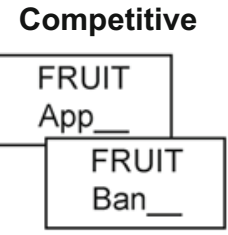

Noncompetitive

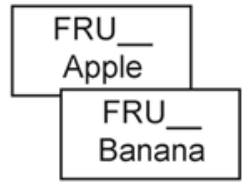

Test

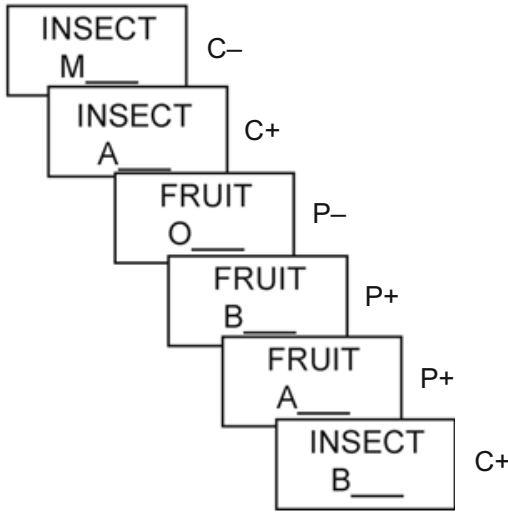

B

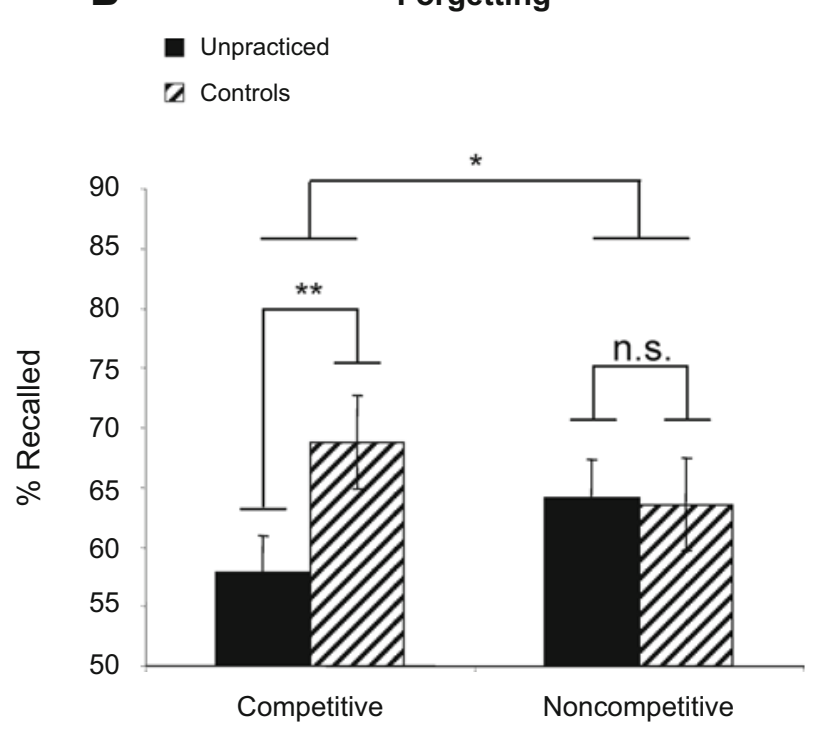

\section{Enhancement}

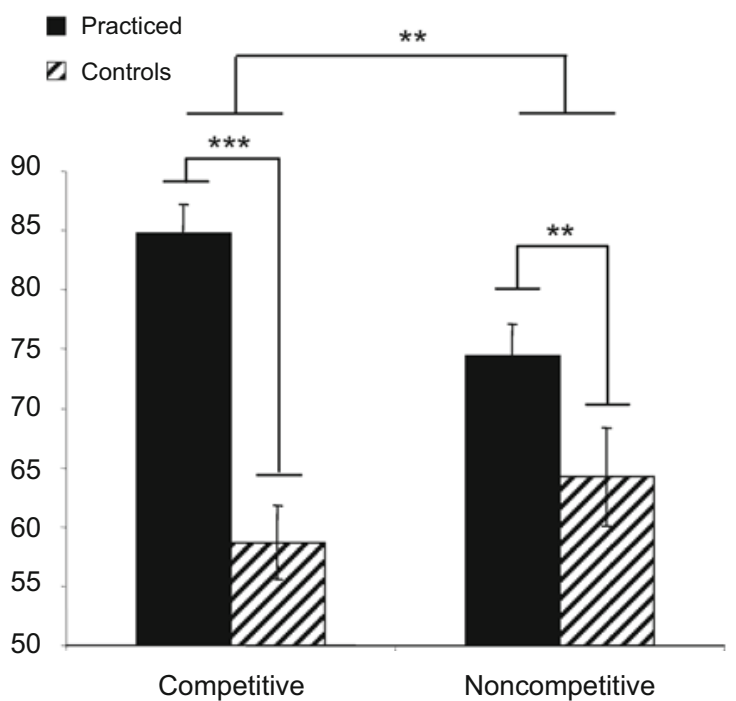

Figure 1. (A) Retrieval practice paradigm as employed in the present study. In the study phase, several items were studied together with their category cues. During the retrieval practice phase, a subset of the prior studied material was repeatedly retrieved. In the competitive condition, the category exemplar was retrieved; in the noncompetitive condition, the category name was retrieved. On a later cued recall test, all previously studied category exemplars were tested. Note that the control items belong to a category of which no items were retrieval practiced. (B) Enhancement and forgetting as induced by prior retrieval practice. Whereas retrieval in the competitive condition induced forgetting, no forgetting was observed in the noncompetitive retrieval condition. In contrast, enhancing effects of retrieval were found in both the competitive and the noncompetitive conditions, although the competitive condition showed stronger enhancement than did the noncompetitive condition. ${ }^{*} p<.05 .{ }^{* *} p<.01 .{ }^{* * *} p<.001$.

cortex in mediating interference and interference resolution in the retrieval practice paradigm. These results are well in line with the view that cognitive interference, in general, is mediated by these brain regions (Botvinick, Braver, Barch, Carter, \& Cohen, 2001).

Going beyond the prior work, the present study examined brain oscillatory activity during competitive memory retrieval. Brain oscillations are rhythmic fluctuations of electrical current in extracellular space that are thought to establish proper communication between distant neural assemblies (Fries, 2005). Such brain oscillations play a crucial role in episodic memory processes, which rely on the coherent (re)activation of a neural network established during encoding (Fuster, 1997). Concerning the brain oscillatory correlates of interference and interference resolution, theta oscillations $(4-7 \mathrm{~Hz})$ in prefrontal brain regions seem to play a crucial role. For instance, in a recent study, we demonstrated that cognitive interference as elicited in a Stroop task is selectively mediated by theta oscillations in the ACC and lateral prefrontal cortex (Hanslmayr et al., 2008). Cavanagh, Cohen, and Allen (2009) extended these findings by demonstrating that prefrontal theta oscillations mediate cognitive interference in an Erikson flanker task. Other studies showed that midfrontal theta oscilla- 
tions increase with the number of items to be held active in working memory and, thus, suggest that theta oscillations also mediate interference in the working memory system (Jensen \& Tesche, 2002; Mecklinger, Kramer, \& Strayer, 1992). Support for the hypothesis that theta oscillations may also mediate interference in the episodic memory system has come from a recently proposed computational neural network model that demonstrated that retrieval competition can be resolved by phase dynamics of theta oscillatory activity (Norman, Newman, \& Detre, 2007). However, whether theta oscillations indeed mediate interference and interference resolution in the episodic memory system has not been investigated empirically to date.

Motivated by the findings described above, we therefore examined whether theta oscillations reflect arising interference and interference resolution during competitive memory retrieval. If so, theta oscillations should increase with increasing retrieval competition and should correlate with interindividual differences in RIF. To address the issue, we followed prior cognitive work (Anderson et al., 2000), in which a competitive retrieval condition was contrasted with a noncompetitive retrieval condition.

Anderson et al. (2000) studied RIF by comparing a competitive retrieval condition, in which the participants were provided with the category name of target material and were asked to retrieve the corresponding item (FRUIT-ban__ ), with a noncompetitive retrieval condition, in which the categorical item was presented intact and subjects were asked to retrieve the category name (FRU___banana). Both conditions were retrieval conditions, with the main difference being that in the competitive condition, there was need to resolve interference from related exemplars, whereas no interference arose in the noncompetitive condition. As would be expected on the basis of the inhibitory account of RIF, forgetting arose in the competitive retrieval condition, but not in the noncompetitive condition. In the present study, we used the noncompetitive retrieval condition as a neural baseline for the competitive retrieval condition. By using such a neural baseline, the present study differed from other recent studies, in which the retrieval practice condition was compared with a reexposure condition (Johansson et al., 2007; Wimber et al., 2009), and permitted control of general retrieval-related activity.

\section{METHOD}

\section{Subjects}

Twenty-four healthy volunteers ( 17 females; mean age, 22.8 years; range, 20-28) participated in the experiment, of whom 3 were lefthanded. All the volunteers were native German speakers, had normal or corrected-to-normal vision, and reported no history of neurological or psychiatric diseases. Prior to the experiment, all subjects gave written informed consent.

\section{Materials}

Two study lists were constructed, each list consisting of words from 18 semantic categories. Each category contained four items that were drawn from two published German word norms (Mannhaupt, 1983; Scheithe \& Bäuml, 1995). For each selected category, the two exemplars with the higher word frequency were used as the not-to-be-practiced competing items (mean rank order of 6.0), and the two exemplars with the lower word frequency were used as the to-be-practiced target items (mean rank order of 10.3). This was done because previous work provided evidence that categories' highfrequency exemplars may be more susceptible to RIF than are categories' low-frequency exemplars (Anderson et al., 1994; Bäuml, 1998). Within each category, no two items began with the same first letter.

\section{Design}

For each subject, the experiment consisted of two blocks. Each block contained three main phases: an initial study phase, an intermediate retrieval practice phase, and a final test phase. The two experimental blocks differed in the list that was provided and the type of condition (competitive or noncompetitive) the subjects performed in the intermediate phase. Following Anderson et al. (2000), the subjects in the competitive condition practiced retrieval of category exemplars, given the category name and the word stem of a target item as a retrieval cue (e.g., FRUIT-ban__ ) ; the subjects in the noncompetitive condition practiced retrieval of category names, given the category's word stem and a target item from the category as a retrieval cue (e.g., FRU___banana). In both conditions, retrieval practice was performed in two third of the categories; the remaining third was left unpracticed and served as a behavioral baseline. This created four types of items: Target items and (unpracticed) competing items from practiced categories are referred to as practiced $(\mathrm{P}+)$ and unpracticed $(\mathrm{P}-)$ items, respectively; target items and competing items from the unpracticed categories served as control items for the $\mathrm{P}+$ and $\mathrm{P}-$ items and are referred to as $\mathrm{C}+$ and $\mathrm{C}-$ items, respectively. Accordingly, the beneficial effect of retrieval practice is defined as the difference between $\mathrm{P}+$ and $\mathrm{C}+$ (target) recall, whereas RIF is defined as the difference between $\mathrm{C}-$ and $\mathrm{P}-$ (competitor) recall. Across subjects, all the targets served equally often as $\mathrm{P}+$ and $\mathrm{C}+$ items, and all competitors served equally often as $\mathrm{P}-$ and $\mathrm{C}-$ items. The presentation order of the lists was counterbalanced across subjects, as was the assignment of list to condition and condition to block.

\section{Procedure}

Study phase. In each of the two experimental blocks, the 72 items of a study list were presented successively on a 15 -in. computer screen, each item together with its category name above it (e.g., FRUIT-banana). The items were shown at a 3 -sec rate with a 1.5 -sec interstimulus interval (blank screen). The subjects were instructed to memorize each item in relation to its category name for an upcoming memory test. Presentation order of the items was randomized within four blocks. Each block consisted of one randomly selected exemplar from each of the 18 categories, with the restriction that a block's last item never belonged to the same category as the next block's first item. The study phase ended with a 30 -sec backward-counting task as a recency control.

Retrieval practice phase. Following the backward-counting task, the subjects engaged in 24 retrieval practice trials (using two nontargets from each of 12 categories). Each practice trial started with a fixation cross with variable duration (1,400-1,600 $\mathrm{msec})$. The subjects were instructed to fixate the cross and to prepare for retrieval practice, avoiding eye movements or blinks. Depending on experimental block, retrieval practice trials were competitive or noncompetitive. In the competitive condition, the subjects were presented the word stem of a target item together with its (complete) category name above it (e.g., FRUIT-ban__ ) and were asked to covertly retrieve the corresponding item from the study list (Johansson et al., 2007; Wimber et al., 2009). In contrast, in the noncompetitive condition, the subjects were provided with a (complete) target item and a category's word stem above it (FRU__banana) and were asked to covertly retrieve the corresponding category name. Each category-item pair was shown for $1.5 \mathrm{sec}$ and was followed by a 1.5 sec blank screen interval, during which the subjects were allowed to blink. Thereafter, the next practice trial was provided. The presentation order of the practice trials was block randomized, analogously to the study phase. After a 3-min distractor task in which the subjects rated the attractiveness of prominent faces, the retrieval practice 
block was repeated for a second time. The retrieval practice phase ended with another 30-sec backward-counting task.

Test phase. Each of the two experimental blocks ended with a cued recall test for all the studied items. Items were tested individually by presenting the item's category name and its unique first letter (e.g., FRUIT- $b_{\_}$). The subjects were given $6 \mathrm{sec}$ per item. The verbal responses were coded by the experimenter as correct, false, or omission. The test was blocked by category, with the restriction that a category's two competitor items $(\mathrm{P}-$ or $\mathrm{C}-)$ were always tested before the category's two target items $(\mathrm{P}+$ or $\mathrm{C}+)$. The order of the categories was counterbalanced such that the mean position of practiced and unpracticed categories was equal across subjects. After a short break, the second block of the experiment was conducted.

\section{EEG Recording}

The EEG was recorded from $64 \mathrm{Ag}-\mathrm{AgCl}$ electrodes mounted in an elastic cap (EASYCAP) positioned according to the extended 10-10 system. The EEG was initially recorded against a reference electrode placed at $\mathrm{FCz}$ and was later rereferenced against a common average reference. Two additional channels placed below and adjacent to the left eye were used to control for horizontal and vertical eye movements and blink artifacts. For data acquisition, a Brainamp MR + amplifier was used (Brain Products, Gilching, Germany), with a sampling rate of $500 \mathrm{~Hz}$. Frequencies between 0.15 and $70 \mathrm{~Hz}$ were recorded with a notch filter at $50 \mathrm{~Hz}$. The EEG data were corrected for eye movements, using calibration data to generate individual artifact coefficients and the algorithm implemented in the software package BESA (MEGIS Software BESA v5.1.8; see Ille, Berg, \& Scherg, 2002, for details). Remaining artifacts, due to muscle activity or poor EOG correction, were excluded by careful visual inspection.

\section{Behavioral Data Analysis}

For behavioral data analysis, two two-way ANOVAs were calculated. To identify the detrimental effects of retrieval practice, an ANOVA with the factors of condition (competitive vs. noncompetitive) and item type (unpracticed vs. controls) was conducted, and to identify the beneficial effects of retrieval practice, an ANOVA with the factors of condition and item type (practiced vs. controls) was conducted. To investigate the relation between the beneficial and detrimental effects of selective retrieval, nonparametric Spearman correlations were calculated.

\section{EEG Analysis}

EEG analysis was carried out using the software package BESA and self-written MATLAB (The MathWorks, Munich, Germany) codes. The EEG data were segmented into 2,000-msec time windows, ranging from $500 \mathrm{msec}$ prior to memory cue presentation to $1,500 \mathrm{msec}$ after memory cue presentation. To get rid of the filter artifacts at the edges of the epochs, the data were filtered in a slightly bigger time interval, but analysis was restricted to the 2,000-msec time window. After artifact rejection, a mean number of 46.4 trials (range: 37-48) remained for the competitive retrieval condition, and a mean number of 45.8 trials (range: $32-48$ ) remained for the noncompetitive retrieval condition.

\section{Analysis of Oscillatory Power}

For analysis of the oscillatory power modulation $\left(\mu \mathrm{V}^{2}\right)$, the Gabor transformation was used, which transforms a signal into a complex time frequency signal, from which the power information can be extracted. The data were filtered in a frequency range of $2-70 \mathrm{~Hz}$. The filter parameter for time frequency resolution (gamma) was set to 1 for the 2 - to $20-\mathrm{Hz}$ frequency range and to pi for the $20-$ to $70-\mathrm{Hz}$ frequency range (see Klimesch, Hanslmayr, Sauseng, Gruber, \& Doppelmayr, 2007, for details). This was done to pick up best the frequency characteristics in each frequency band (comparable to a morlet-wavelet transformation, which also changes time frequency resolution to accommodate for the different frequency characteristics). To quantify event-related power changes, the percentage of poststimulus power change in relation to a prestimulus baseline period was calculated. The baseline period was set to $-500 \mathrm{msec}$ to stimulus onset. For statistical analysis, the signal change values were collapsed in order to obtain six frequency bands, theta (4-7 Hz), alpha $(8-12 \mathrm{~Hz})$, beta $_{1}(13-19 \mathrm{~Hz})$, beta $2(20-30 \mathrm{~Hz})$, gamma $_{1}(35-45 \mathrm{~Hz})$, and gamma $2(55-70 \mathrm{~Hz})$, and to obtain three time windows, $\mathrm{T}_{1}(0-500 \mathrm{msec}), \mathrm{T}_{2}(500-1,000 \mathrm{msec})$, and $\mathrm{T}_{3}$ $(1,000-1,500 \mathrm{msec})$.

\section{Analysis of Phase Locking}

Changes in oscillatory power can be due to induced responses, not phase locked to stimulus onset, or to evoked responses, which are phase locked to stimulus onset (Hanslmayr, Klimesch, et al., 2007; Herrmann, Munk, \& Engel, 2004). To investigate such evoked oscillatory dynamics, phase locking was examined by computing the phase-locking index (PLI; Gruber, Klimesch, Sauseng, \& Doppelmayr, 2005; Tallon-Baudry, Bertrand, Delpuech, \& Permier, 1996). The PLI is a measure of phase consistency across single trials at a given frequency for each time point. The PLI ranges from 0 , which means no phase locking at all, to 1 , which means perfect phase locking. To obtain phase estimates for each single trial, frequency band, and time point, the Gabor transformation was used, which permits extraction of the phase information (see Gruber et al., 2005, for details). The same filter parameters as those for the power analysis were used. For statistical analysis, the same frequency bands as those for the power analysis were used. Because evoked oscillatory responses are most prominent in the early time period, phase locking was investigated only in the 0 - to 500 -msec time interval.

\section{Analysis of ERP Waveforms}

To further investigate the evoked effects, the ERP waveforms were analyzed. The ERPs were computed for each subject and condition in a time window ranging from -100 to $1,500 \mathrm{msec}$. The waveforms were low-pass filtered at $15 \mathrm{~Hz}$ (48 db/oct roll-off). After inspection of the grand-average waveforms, three time windows were used for analysis: 200-250, 300-350, and 360-400 msec (see Figure 3), where strong differences between the competitive and the noncompetitive condition arose. The mean amplitudes in these three time windows were then subjected to a statistical randomization procedure (see below).

\section{Statistical Analysis}

For statistical analysis, nonparametric randomization tests were used, employing a two-stage randomization procedure to account for multiple testing. This procedure is based on the method described by Blair and Karniski (1993) and had already been applied in several other studies (Bäuml, Hanslmayr, Pastötter, \& Klimesch, 2008; Hanslmayr, Aslan, et al., 2007; Hanslmayr, Spitzer, \& Bäuml, 2009). The procedure works as follows. First, pairwise comparisons between the competitive and the noncompetitive conditions were conducted using Wilcoxon sign-rank tests. These tests were computed for each electrode position and revealed the number of electrodes that showed a significant effect ( $p<.05$, two-tailed). Second, a randomization test using 2,000 permutation runs was conducted. In this procedure, the two conditions were interchanged randomly for each participant and each randomization run, thereby eliminating any systematic difference between the two conditions. After each randomization run, Wilcoxon sign-rank tests were calculated, returning the number of electrodes showing a significant difference between the two conditions. After 2,000 such permutation runs, a random distribution of the number of electrodes showing a significant difference between the two conditions was generated. This distribution was then used to determine the $p$ level of an effect, reflecting how many times a given number of significant electrodes (e.g., eight) was exceeded during the permutation runs (values higher than eight). Thus, the $p$ level reflects the probability that a given number of electrodes exhibiting a significant difference between two conditions would be found by chance. As in a previous study (Hanslmayr, Spitzer, \& Bäuml, 2009), frontal electrode sites were excluded from the randomization test in 


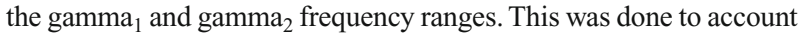
for possible contamination of miniature saccades, which give rise to frontal gamma effects if an average reference is employed (YuvalGreenberg, Tomer, Keren, Nelken, \& Deouell, 2008).

\section{RESULTS}

\section{Behavioral Results}

A depiction of the behavioral results can be found in Figure 1B. Concerning the forgetting effect of selective retrieval, the two-way ANOVA revealed a significant item type $\times$ condition interaction $[F(1,23)=7.12, p<.05]$. This interaction was due to a robust RIF effect in the competitive condition [P- items, $57.8 \%$; control items, $68.8 \%$; $t(23)=3.52, p<.005]$, whereas no forgetting was evident in the noncompetitive condition ( $\mathrm{P}-$ items, 64.1\%; control items, $63.5 \% ; p>.5)$. In addition, $\mathrm{P}-$ items in the competitive condition were recalled less accurately than $\mathrm{P}-$ items in the noncompetitive condition $[t(23)=$ $2.46, p<.05]$. The main effect for condition did not reach significance $(p>.5)$.

Concerning the enhancement effect of selective retrieval, a significant item type $\times$ condition interaction was obtained $[F(1,23)=13.14, p<.005]$, showing that retrieval in the competitive condition induced stronger enhancement $[\mathrm{P}+$ items, 84.7\%; control items, 58.7\%; $t(23)=10.07$, $p<.001]$ than did retrieval in the noncompetitive condition $[\mathrm{P}+$ items, $74.5 \%$; control items, $64.2 \%$; $t(23)=3.43$, $p<.005]$. Moreover, $\mathrm{P}+$ items in the competitive condition were recalled better than $\mathrm{P}+$ items in the noncompetitive condition $[t(23)=3.56, p<.005]$. A correlation analysis across subjects revealed that the amount of retrieval-induced enhancement (practiced - control) and the amount of RIF (unpracticed - control) in the competitive condition were not related to each other $(r=-.13, p>.5)$. An additional analysis showed that the same forgetting and enhancement effects were obtained when the baseline items were pooled across the competitive and noncompetitive conditions. ${ }^{1}$

\section{EEG Results}

Power. An overview of the statistical results of oscillatory power is shown in Table 1 . The table shows that a significant effect emerged in the early time window $(0-$ $500 \mathrm{msec}$ ) in the theta frequency band only. The time frequency plot in Figure 2A plots the difference between the competitive and the noncompetitive conditions for a pool of left parietal electrode sites. The early increase in theta power was stronger in the competitive than in the noncompetitive condition $\left(p_{\text {corr }}<.05\right.$; see Figure $\left.2 \mathrm{~B}\right)$. This increase was evident over left-parietal and frontocentral electrode sites, although only the left-parietal electrode sites exceeded the statistical threshold (Figure 2C). No significant effects were found in the other frequency bands. In the alpha and

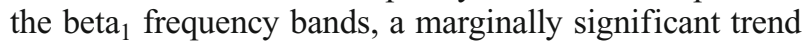
$\left(p_{\text {corr }}<.1\right)$ was found in the middle $(500-1,000 \mathrm{msec})$ and late $(1,000-1,500 \mathrm{msec})$ time windows, reflecting a slightly stronger power decrease for the competitive than for the noncompetitive retrieval condition (Table 1).

Phase locking. To investigate whether the early theta amplitude increase was due to evoked or induced oscilla-
Table 1

Number of Electrodes Showing Significant Differences

Between Competitive and Noncompetitive Retrieval

\begin{tabular}{lccccccc}
\hline & \multicolumn{3}{c}{$\begin{array}{c}\text { Comp. }> \\
\text { Noncomp. }\end{array}$} & & \multicolumn{3}{c}{$\begin{array}{c}\text { Comp. } \\
\text { Noncomp. }\end{array}$} \\
\cline { 2 - 4 } \cline { 5 - 8 } & T1 & T2 & T3 & & T1 & T2 & T3 \\
\hline Theta & $9^{*}$ & - & - & & - & 1 & - \\
Alpha & - & - & - & & - & 3 & $6^{+}$ \\
Beta $_{1}$ & - & - & - & & - & $4^{+}$ & 3 \\
Beta $_{2}$ & 2 & 3 & - & & - & 2 & 2 \\
Gamma $_{1}$ & 2 & 1 & - & 1 & 1 & - \\
Gamma $_{2}$ & 2 & 1 & - & 3 & - & - \\
\hline
\end{tabular}

${ }^{+} p_{\text {corr }}<.1 . \quad{ }^{*} p_{\text {corr }}<.05$.

tory activity, phase locking across single trials was calculated by means of the PLI (Figure 2D). The results showed that theta phase locking was stronger in the competitive than in the noncompetitive condition $\left(p_{\text {corr }}<.001\right.$; Figure $2 \mathrm{E}$ ), with this increase being pronounced over leftparietal and frontocentral electrode sites (Figure 2F). These results demonstrate that the early increase in theta amplitude was due to evoked oscillatory activity. ${ }^{2}$ In the beta $_{1}$ frequency range (13-19 Hz), a higher PLI was observed in the competitive condition than in the noncompetitive condition $\left(p_{\text {corr }}<.001\right.$; Figure 2D). This effect was evident from 0 to $400 \mathrm{msec}$ with a left-parietal and right-frontal topography (Supplemental Figure S1).

\section{ERP Results}

The results of the ERP analysis are plotted in Figure 3. As is suggested by the phase-locking analysis, evoked activity in the theta frequency range can be seen in the ERPs over parietal and frontocentral electrode sites. A first effect was obtained around $225 \mathrm{msec}$, where the competitive condition showed a stronger negativity over parietal electrode sites and a stronger positivity over frontocentral electrode sites than did the noncompetitive condition (Figure 3C). A second effect was evident $100 \mathrm{msec}$ later, where the competitive condition showed a stronger positivity at parietal electrode sites (Figure 3D). A third effect was obtained around $380 \mathrm{msec}$, where a positive component emerged over frontocentral electrode sites, reflecting a stronger positivity for the competitive than for the noncompetitive retrieval condition (Figure 3E). Similar results were obtained for the current source density transformed data, showing that these effects are independent of the average reference method (Supplemental Figure S2).

\section{Relation Between EEG and Behavior}

To examine the relationship between the brain oscillatory differences and the behavioral effects of selective memory retrieval, nonparametric correlations were computed. For this analysis, the difference between the competitive and noncompetitive conditions was pooled across those electrode sites that revealed a significant effect in the prior amplitude and PLI analysis. As is shown in Figure 4, the early theta PLI increase $(0-500 \mathrm{msec})$ was negatively correlated with forgetting ${ }^{3}(r=-.62, p<.001)$. No correlation was found between theta PLI and enhancement $(r=.29, p>.15)$. Both correlation coefficients differed 
A Competitive-Noncompetitive

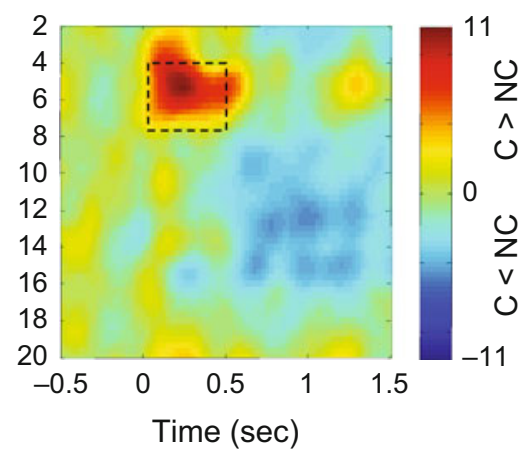

D Competitive - Noncompetitive

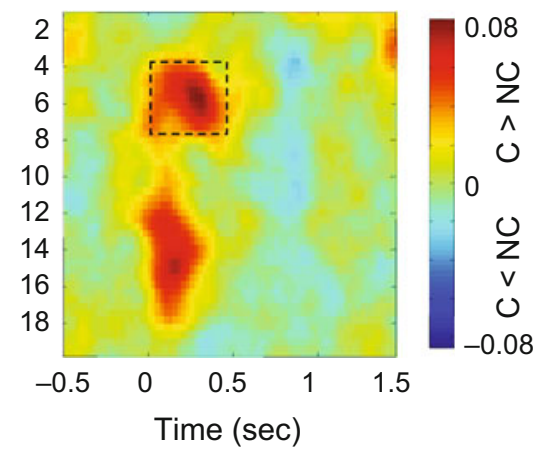

B

$\theta$ - Power

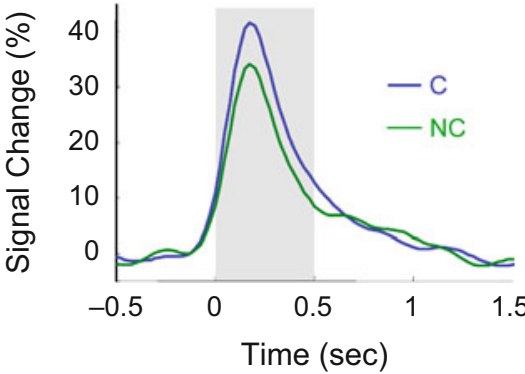

E

$$
\theta-\mathrm{PLI}
$$

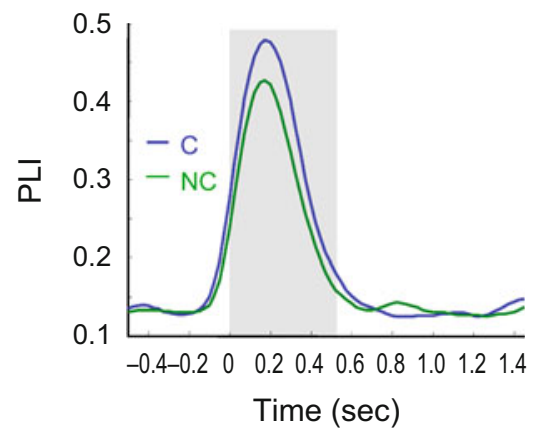

C

$\theta$ - Power

Figure 2. Results in the theta frequency band. (A) Time frequency plot showing the difference in oscillatory power between the competitive (C) and the noncompetitive (NC) conditions, plotted for those left-parietal electrodes that exhibited a significant difference (C vs. NC). Stronger power in the $C$ condition is coded with warm colors; weaker power in the $C$ condition is coded with cold colors. (B) Time course of theta power in the $\mathrm{C}$ and NC conditions. The gray bar indicates the time window during which a significant difference between the two conditions was observed $\left(p_{\text {corr }}<.05\right)$. (C) Topography of the theta power difference in the early time window between the $\mathrm{C}$ and the NC conditions. The right plot shows the significant electrode sites, obtained by nonparametric Wilcoxon signrank tests. (D) Plot of the results of the phase-locking analysis. The time frequency plot shows the phase-locking index (PLI) difference, pooled over frontocentral and left-parietal electrode sites, between the $\mathrm{C}$ and the NC conditions. Warm colors indicate more phase locking in the $C$ condition. (E) Time course of the theta PLI. (F) Topography of the theta PLI difference between the C and the NC conditions. The right plot shows the electrode sites that exhibited significantly stronger phase locking in the $\mathrm{C}$ than in the $\mathrm{NC}$ condition.

significantly from each other $(Z=3.10, p<.001)$. No significant correlation between theta power and forgetting was observed $(p>.5)$.

No significant correlation was obtained between the beta $_{1}$ PLI and behavior (both forgetting and enhancement; $p \mathrm{~s}>$.2). Also, no significant correlations with behavior were obtained for the ERP effects (competitive - noncompetitive) in any of the three time windows (200-250, $300-350$, and $360-400 \mathrm{msec} ; p \mathrm{~s}>.10$ ).

\section{DISCUSSION}

On a behavioral level, our results replicate prior cognitive work in showing that competitive retrieval practice causes forgetting of related but unpracticed memory items, whereas noncompetitive retrieval practice does not induce forgetting (Anderson et al., 2000). These results are consistent with the inhibition account of RIF, which assumes that related items interfere during retrieval practice and are inhibited to reduce the interference (e.g., Anderson, 2003). The results also confirm that the noncompetitive retrieval condition can be used as a neural baseline for the competitive retrieval condition, to isolate the interferenceresolving mechanism and control for general retrievalrelated neural activity.

Concerning brain oscillations, the present study shows that competitive memory retrieval induces higher levels of evoked theta activity than does retrieval in a noncompetitive condition. In addition, the competition-related increase in theta oscillatory activity predicted the amount of subsequent forgetting. The present study complements previous studies exploring the neural foundations of RIF by analyzing ERPs (Johansson et al., 2007) and fMRI (Kuhl et al., 2007; Wimber et al., 2008) and is the first one to show that interference and interference resolution during episodic memory retrieval is selectively reflected in theta oscillatory activity.

\section{Relation to Prior Interference and RIF Studies}

There is broad agreement in the literature that RIF arises due to interference from competing memory items (e.g., Anderson, 2003; Bäuml et al., 2010) and reliably triggers neural processes in the prefrontal cortex, reflecting arising interference and interference resolution (Kuhl et al., 
A

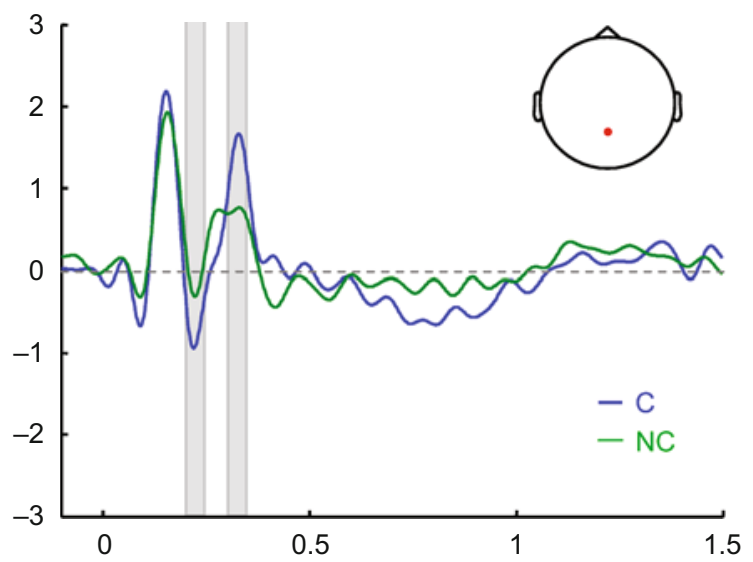

B

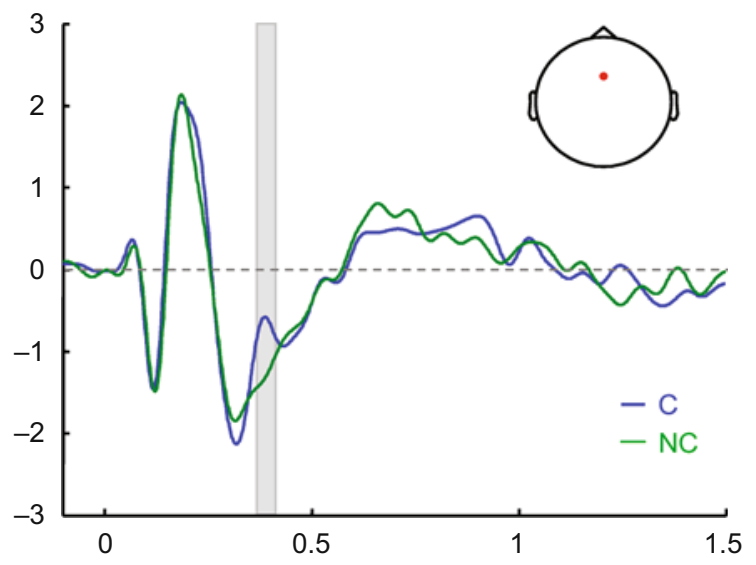

C 200-250 msec

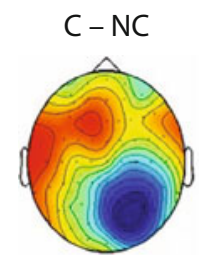

$\mu \mathrm{V}$

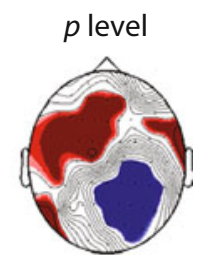

$$
p_{\text {corr }}<.001
$$

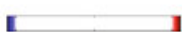

D

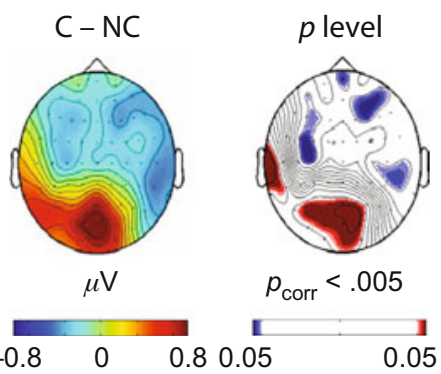

E

360-400 msec

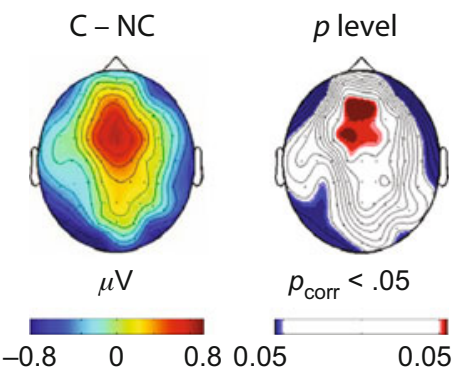

Figure 3. Plot of the results of the ERP analysis. (A) Grand average ERPs of the competitive (C) and the noncompetitive (NC) conditions for a midparietal electrode (Pz). (B) Grand average ERPs for the $\mathrm{C}$ and the NC conditions plotted for a frontocentral electrode site $(\mathrm{Fz})$. The gray bars indicate the time windows where significant differences emerged ( $\left.p_{\text {corr }}<.05\right)$. (C-E) Plots of the topography for the effects in the (C) 200- to 250-msec, (D) 300- to 350-msec, and (E) 360- to 400-msec time windows. Warm colors indicate stronger positivity for the $\mathbf{C}$ condition. Cold colors indicate stronger negativity for the $\mathbf{C}$ condition. The left plots indicate the topographical distribution of the amplitude; the right plots indicate the topography of the $p$ level (obtained by nonparametric Wilcoxon tests).

2007; Wimber et al., 2009). Several previous studies demonstrated that theta oscillations vary as a function of interference in a cognitive task. Mecklinger et al. (1992), for instance, showed that theta power over frontocentral electrodes increases with the number of items held in working memory during retrieval (for similar findings, see Jensen $\&$ Tesche, 2002). Although, in principle, these effects could be attributed to task effort or difficulty, increased theta oscillations could also reflect the number of stored items in memory and, thereby, memory interference. Indeed, other studies demonstrated that prefrontal theta power and phase coupling increase as a function of interference in response conflict tasks (Cavanagh et al., 2009; Hanslmayr et al., 2008). On the basis of these findings, the present result of increased theta oscillations in the competitive retrieval condition likely reflects generally higher levels of interference in competitive than in noncompetitive retrieval.

On an interindividual level, a strong negative correlation arose between theta oscillatory activity and amount of RIF. The inhibitory account of RIF assumes that interference triggers inhibitory processes, which resolve the interference by directly affecting the interfering items' memory representations (Anderson \& Spellman, 1995; Spitzer \&
Bäuml, 2007; Veling \& van Knippenberg, 2004). Following this account, the observed individual level of theta activity may represent the net result of arising interference and subsequent interference resolution. In this case, high degrees of interference resolution may lead to low resulting levels of interference, due to efficient inhibition of the competing memory items and high degrees of forgetting, which is exactly what the present relationship between theta oscillatory activity and RIF suggests. If frontal midline theta oscillations are generated in the ACC (Ishii et al., 1999; Onton, Delorme, \& Makeig, 2005; Sauseng, Hoppe, Klimesch, Gerloff, \& Hummel, 2007), our results are in accordance with a recent fMRI study, demonstrating a negative correlation between ACC activation and RIF (Wimber et al., 2009). This suggestion is also in line with the results of Kuhl et al. (2007), who showed that the BOLD signal in prefrontal brain regions decreased over repeated retrieval attempts and that this decrease predicted RIF.

The evoked theta effect in the present study showed a frontocentral and parietal topography, whereas the effect in theta power was evident only over left-parietal electrode sites. Importantly, however, the left-parietal theta power increase was not correlated with later forgetting, whereas 


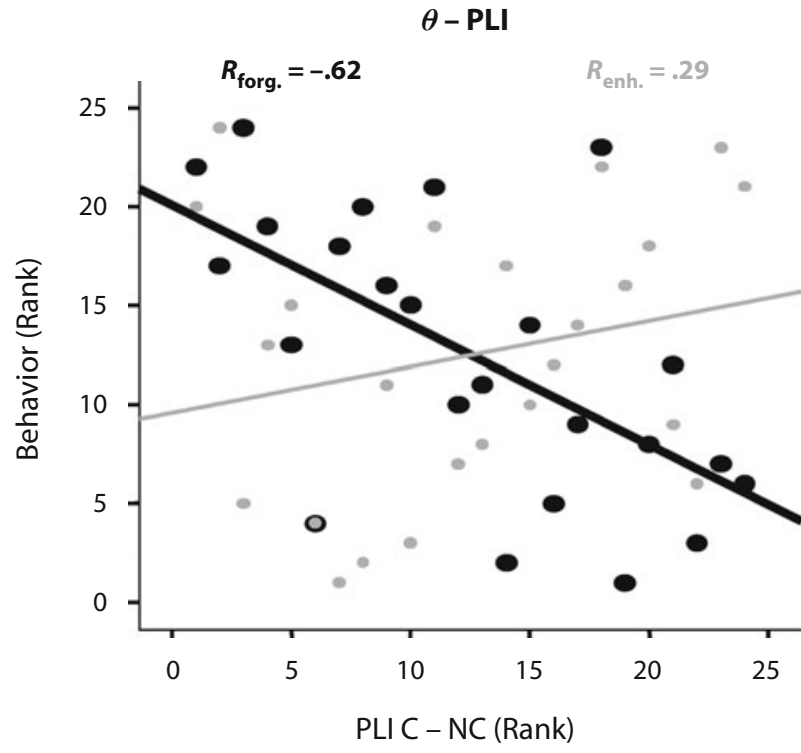

Figure 4. Plot of the brain-behavior correlation results. The scatterplot depicts the rank correlation of theta phase-locking index (PLI) difference (competitive [C] - noncompetitive [NC]) with forgetting (black dots) and enhancement (gray dots). The values for theta phase locking were averaged over those frontal and parietal electrode sites that exhibited a significant difference between the $\mathrm{C}$ and the NC conditions (see Figure 2F).

theta phase locking over both parietal and frontocentral electrodes predicted RIF (see note 3). Such a topographical pattern during memory retrieval has been described previously in studies examining ERPs (Rugg \& Curran, 2007) and theta oscillations (Jacobs, Hwang, Curran, \& Kahana, 2006) and may indicate a memory-retrievalspecific process. The pattern is also consistent with recent fMRI studies showing an involvement of parietal and prefrontal cortical areas in selective memory retrieval (Kuhl et al., 2007; Wimber et al., 2009). The neural sources that gave rise to frontocentral and parietal phase locking, however, are hard to assess from EEG data, and whether phase modulations can be seen in the BOLD signal is unclear to date. These questions might be addressed in future studies, possibly combining EEG and fMRI recording.

\section{Alternative Interpretations}

A general objection that can be raised against the interpretation of our results is that retrieval in the competitive condition (FRUIT-app__) was much more difficult than retrieval in the noncompetitive condition (FRU_-apple). The finding of increased theta oscillatory activity in the competitive retrieval condition could thus reflect increased retrieval effort, instead of retrieval competition. However, there are several reasons that render this alternative explanation unlikely. First, prior studies examining theta oscillations at retrieval showed decreased levels of theta activity for memory items that are difficult to retrieve (Klimesch et al., 2006; Spitzer, Hanslmayr, Opitz, Mecklinger, \& Bäuml, 2009). For instance, early evoked theta oscillations decrease with increasing lag between encoding and retrieval (Klimesch et al., 2006) and are lower for inhibited memory items (Spitzer et al., 2009), indicating that theta oscillations are not positively related to retrieval effort. Second, the retrieval effort explanation of our results does not explain the present finding that theta oscillations predict the interindividual differences of RIF.

Another possibility is that the observed theta effect is due to priming processes, since the category name was retrieved twice in the noncompetitive condition, whereas the memory item was retrieved only once in the competitive condition. This alternative explanation, however, cannot explain the observed negative correlation between theta phase locking and RIF.

\section{Implications for Accounts of RIF}

Although it is widely assumed that RIF is caused by inhibition (e.g., Anderson, 2003; Bäuml et al., 2010), an alternative account of RIF suggests that selective retrieval leads to forgetting of competing memories by means of blocking (Camp, Pecher, \& Schmidt, 2007; Williams \& Zacks, 2001). This account assumes that during the final test phase, the strong, retrieval-practiced items block access to the weak, unpracticed items. The results of the present study are inconsistent with this account. On a behavioral level, the blocking account predicts that the amount of enhancement and the amount of forgetting should be correlated, which was not the case (for related results, see Anderson et al., 2000; Bäuml \& Kuhbandner, 2007; Román, Soriano, Gomez-Ariza, \& Bajo, 2009). On an electrophysiological level, the finding that theta oscillations selectively predict forgetting but not enhancement is inconsistent with blocking, which would predict that enhanced theta activity should also correlate with enhancement. Our finding is in line with prior fMRI and EEG studies, also reporting dissociable neural correlates of enhancement and forgetting in the retrieval practice paradigm (Kuhl et al., 2007; Spitzer et al., 2009; Wimber et al., 2008).

Interestingly, the reported electrophysiological effects of arising interference and interference resolution appeared quite early, peaking around $250 \mathrm{msec}$ after stimulus onset. The evoked theta oscillatory effect was also evident in the ERP, reflected by a series of ERP peaks with an interpeak latency of around $100 \mathrm{msec}(225-325 \mathrm{msec})$ and $150 \mathrm{msec}(225-380 \mathrm{msec})$ and a frontocentral and parietal topography. This result demonstrates that the evoked theta oscillatory effect was not due to a single ERP component being higher in the competitive condition, thus suggesting the action of an evoked oscillatory mechanism. The finding of an early theta effect is consistent with the results of a previous study (Rizzuto, Madsen, Bromfield, SchulzeBonhage, \& Kahana, 2006), showing that evoked theta oscillations, recorded within the inferior temporal cortex, mediate memory retrieval as early as $100-500 \mathrm{msec}$ after stimulus onset. Similarly, several ERP studies have shown early retrieval-related activity, starting around $200 \mathrm{msec}$ after presentation of the memory cue (Friedman \& Johnson, 2000). Together with these findings, the present results suggest that memory retrieval and, with it, the interference-resolving mechanisms are reflected in early evoked theta oscillations. It should be noted, however, that in order to conform to the standard behavioral 
paradigm, a blocked design was employed in the present study. The early effect therefore may be affected by tonic activity, being present throughout the competitive retrieval block. Further studies are required to confirm these early retrieval effects, varying competitive and noncompetitive retrieval on a trial-by-trial basis.

Apart from the indirect measure of inhibition, which arose from the negative correlation between theta phase locking and forgetting, we did not identify a physiological mechanism reflecting active inhibition of the interfering material. Such a mechanism would implicate electrophysiological activity's showing a positive correlation with forgetting. On the basis of recent evidence suggesting that an increase in alpha oscillations can reflect top-down-driven inhibition (Klimesch, Sauseng, \& Hanslmayr, 2007), one might have expected to find increased alpha power during competitive retrieval to predict later forgetting. In line with such a top-down inhibitory view, a recent study showed that directed forgetting, a paradigm in which subjects are cued to intentionally forget previously studied information, is mediated selectively by alpha oscillations (Bäuml et al., 2008). RIF, however, differs from directed forgetting in at least two important ways: RIF is unintentional and operates on an item level, whereas directed forgetting is intentional and operates on a list level (e.g., Kimball \& Bjork, 2002). Together with the results from the Bäuml et al. (2008) study, the present results therefore suggest that alpha oscillations might be involved only if inhibitory mechanisms are triggered intentionally and/or operate on a list level. Future work examining episodic forgetting in paradigms in which forgetting is intentional and occurs on an item level (e.g., think/no-think paradigm; Anderson \& Green, 2001; Bergström, de Fockert, \& RichardsonKlavehn, 2009; Hanslmayr, Leipold, Pastötter, \& Bäuml, 2009) thus might improve our understanding of the role of alpha oscillations for episodic inhibition.

\section{Conclusions}

The present results suggest that theta oscillations reflect interference and interference resolution during episodic memory retrieval. Thereby, the early evoked theta effect may reflect (1) generally higher levels of interference in the competitive than in the noncompetitive retrieval condition and (2) the effects of interference resolution by inhibition, which is suggested by the negative correlation between theta phase locking and the amount of RIF. Although our results are well in line with the cognitive theory of RIF (Anderson, 2003; Bäuml et al., 2010), no direct measure of the inhibitory mechanism was obtained. Thus, the question of whether the reduction in early evoked theta oscillations reflects such an inhibitory mechanism remains to be explored in future studies. Besides, the findings are consistent with the results from prior work examining oscillatory interference effects in other cognitive domains and converge on the view that, concerning brain oscillatory correlates of interference and interference resolution, theta oscillations play a crucial role.

\section{AUTHOR NOTE}

The authors thank Verena Bauer and Anna-Sofie Kunz for their help with data acquisition. The authors also thank Maria Wimber and Bern- hard Pastötter for their helpful comments on previous versions of the manuscript. Correspondence concerning this article should be addressed to S. Hanslmayr, Department of Psychology, University of Konstanz, 78457 Konstanz, Germany (e-mail: simon.hanslmayr@uni-konstanz .de).

\section{REFERENCES}

Anderson, M. C. (2003). Rethinking interference theory: Executive control and the mechanisms of forgetting. Journal of Memory \& Language, 49, 415-445.

Anderson, M. C., BJork, E. L., \& BJork, R. A. (2000). Retrievalinduced forgetting: Evidence for a recall-specific mechanism. Psychonomic Bulletin \& Review, 7, 522-530.

Anderson, M. C., BJork, R. A., \& BJork, E. L. (1994). Remembering can cause forgetting: Retrieval dynamics in long-term memory. Journal of Experimental Psychology: Learning, Memory, \& Cognition, 20, 1063-1087.

Anderson, M. C., \& Green, C. (2001). Suppressing unwanted memories by executive control. Nature, 410, 366-369.

Anderson, M. C., \& NeEly, J. H. (1996). Interference and inhibition in memory retrieval. In E. L. Bjork \& R. A. Bjork (Eds.), Memory: Handbook of perception and cognition (pp. 237-313). San Diego: Academic Press.

Anderson, M. C., \& Spellman, B. A. (1995). On the status of inhibitory mechanisms in cognition: Memory retrieval as a model case. Psychological Review, 102, 68-100.

BäUML, K.-H. (1998). Strong items get suppressed, weak items do not: The role of item strength in output interference. Psychonomic Bulletin \& Review, 5, 459-463.

BäUML, K.-H. (2002). Semantic generation can cause forgetting. Psychological Science, 13, 357-361.

BÄUML, K.-H. (2008). Inhibitory processes. In H. L. Roediger III (Ed.), Cognitive psychology of memory (pp. 195-220). Oxford: Elsevier.

BäUmL, K.-H., \& Aslan, A. (2004). Part-list cuing as instructed retrieval inhibition. Memory \& Cognition, 32, 610-617.

Bäuml, K.-H., Hanslmayr, S., Pastötter, B., \& Klimesch, W. (2008). Oscillatory correlates of intentional updating in episodic memory. NeuroImage, 41, 596-604.

Bäuml, K.-H., \& Kuhbandner, C. (2007). Remembering can cause forgetting - but not in negative moods. Psychological Science, 18, 111-115.

Bäuml, K.-H., Pastötter, B., \& Hanslmayr, S. (2010). Binding and inhibition in episodic memory-Cognitive, emotional, and neural processes. Neuroscience \& Biobehavioral Reviews, 34, 1047-1054. doi:10.1016/j.neubiorev.2009.04.005

Bergström, Z., de Fockert, J. W., \& Richardson-Klavehn, A. (2009). ERP and behavioral evidence for direct suppression of unwanted memories. NeuroImage, 48, 726-737.

BlAIR, R. C., \& KARNISKI, W. (1993). An alternative method for significance testing of waveform difference potentials. Psychophysiology, 30, 518-524.

Botvinick, M. M., Braver, T. S., Barch, D. M., Carter, C. S., \& Cohen, J. D. (2001). Conflict monitoring and cognitive control. Psychological Review, 108, 624-652.

CAmp, G., Pecher, D., \& Schmidt, H. G. (2007). No retrieval-induced forgetting using item-specific independent cues: Evidence against a general inhibitory account. Journal of Experimental Psychology: Learning, Memory, \& Cognition, 33, 950-958.

Cavanagh, J. F., Cohen, M. X., \& Allen, J. J. (2009). Prelude to and resolution of an error: EEH phase synchrony reveals cognitive control dynamics during action monitoring. Journal of Neuroscience, 29, 98-105.

Ciranni, M. A., \& Shimamura, A. P. (1999). Retrieval-induced forgetting in episodic memory. Journal of Experimental Psychology: Learning, Memory, \& Cognition, 25, 1403-1414.

Friedman, D., \& JoHnson, R. (2000). Event-related potential (ERP) studies of memory encoding and retrieval: A selective review. Microscopy Research \& Technique, 51, 6-28.

FrIES, P. (2005). A mechanism for cognitive dynamics: Neural communication through neuronal coherence. Trends in Cognitive Sciences, 9, 474-480.

Fuster, J. M. (1997). Network memory. Trends in Neurosciences, 20, 451-459. 
Gruber, W. R., Klimesch, W., Sauseng, R., \& Doppelmayr, M. (2005). Alpha phase synchronization predicts P1 and N1 latency and amplitude size. Cerebral Cortex, 15, 371-377.

Hanslmayr, S., Aslan, A., Staudigl, T., Klimesch, W., Herrmann, C. S., \& BäUML, K.-H. (2007). Prestimulus oscillations predict visual perception performance between and within subjects. NeuroImage, 37, 1465-1473.

Hanslmayr, S., Klimesch, W., Sauseng, P., Gruber, W., DoppelMAYr, M., Freunberger, R., ET AL. (2007). Alpha phase reset contributes to the generation of ERPs. Cerebral Cortex, 17, 1-8.

Hanslmayr, S., Leipold, P., Pastötter, B., \& BäUmL, K.-H. (2009). Anticipatory signatures of voluntary memory suppression. Journal of Neuroscience, 29, 2742-2747.

Hanslmayr, S., Pastötter, B., Bäuml, K.-H., Gruber, S., WimBER, M., \& KLIMESCH, W. (2008). The electrophysiological dynamics of interference during the Stroop task. Journal of Cognitive Neuroscience, 20, 215-225.

Hanslmayr, S., Spitzer, B., \& BäUml, K.-H. (2009). Brain oscillations dissociate between semantic and nonsemantic encoding of episodic memories. Cerebral Cortex, 19, 1631-1640.

Herrmann, C. S., Munk, M. H. J., \& Engel, A. K. (2004). Cognitive functions of gamma-band activity: Memory match and utilization. Trends in Cognitive Sciences, 8, 347-355.

Ille, N., Berg, P., \& Scherg, M. (2002). Artifact correction of the ongoing EEG using spatial filters based on artifact and brain signal topographies. Journal of Clinical Neurophysiology, 19, 113-124.

IshiI, R., Shinosaki, K., Ukai, S., Inouye, T., Ishihara, T., \& Yoshimine, T. (1999). Medial prefrontal cortex generates frontal midline theta rhythm. NeuroReport, 10, 675-679.

Jacobs, J., Hwang, G., Curran, T., \& Kahana, M. J. (2006). EEG oscillations and recognition memory: Theta correlates of memory retrieval and decision making. NeuroImage, 32, 978-987.

Jensen, O., \& Tesche, C. D. (2002). Frontal theta activity in humans increases with memory load in a working memory task. European Journal of Neuroscience, 15, 1395-1399.

Johansson, M., Aslan, A., Bäuml, K.-H., Gäbel, A., \& MecKLINGER, A. (2007). When remembering causes forgetting: Electrophysiological correlates of retrieval-induced forgetting. Cerebral Cortex, 17, 1335-1341.

Kimball, D. R., \& BJoRK, R. A. (2002). Influences of intentional and unintentional forgetting on false memories. Journal of Experimental Psychology: General, 131, 116-130.

Klimesch, W., Hanslmayr, S., Sauseng, P., Gruber, W., Brozinsky, C. J., Kroll, N. E. A., ET AL. (2006). Oscillatory EEG correlates of episodic trace decay. Cerebral Cortex, 16, 280-290.

Klimesch, W., Hanslmayr, S., Sauseng, P., Gruber, W. R., \& DopPELMAYr, M. (2007). P1 and traveling alpha waves: Evidence for evoked oscillations. Journal of Neurophysiology, 97, 1311-1318.

Klimesch, W., Sauseng, P., \& Hanslmayr, S. (2007). EEG alpha oscillations: The inhibition-timing hypothesis. Brain Research Reviews, 53, 63-88.

Kuhl, B., Dudukovic, N. M., Kahn, I., \& Wagner, A. D. (2007). Decreased demands on cognitive control reveal the neural processing benefits of forgetting. Nature Neuroscience, 10, 908-914.

Mannhaupt, H. R. (1983). Reproduktionsnormen für verbale Reaktionen zu 40 geläufigen Kategorien. Sprache \& Kognition, 4, 264-278.

Mecklinger, A., Kramer, A. F., \& Strayer, D. L. (1992). Event related potentials and EEG components in a semantic memory search task. Psychophysiology, 29, 104-119.

Norman, K. A., Newman, E. L., \& Detre, G. (2007). A neural network model of retrieval-induced forgetting. Psychological Review, 114, 887-953.

Onton, J., Delorme, A., \& Makeig, S. (2005). Frontal midline EEG dynamics during working memory. NeuroImage, 27, 341-356.

Rizzuto, D. S., Madsen, J. R., Bromfield, E. B., SchulzeBonhage, A., \& Kahana, M. J. (2006). Human neocortical oscillations exhibit theta phase differences between encoding and retrieval. NeuroImage, 31, 1352-1358.

Román, P., Soriano, M. F., Gomez-Ariza, C. J., \& BaJo, M. T. (2009). Retrieval-induced forgetting and executive control. Psychological Science, 20, 1053-1058.

RugG, M. D., \& Curran, T. (2007). Event-related potentials and recognition memory. Trends in Cognitive Sciences, 11, 251-257.
Sauseng, P., Hoppe, J., Klimesch, W., Gerloff, C., \& Hummel, F. (2007). Dissociation of sustained attention from central executive functions: Local activity and interregional connectivity in the theta range. European Journal of Neuroscience, 25, 587-593.

SCHEITHe, K., \& BÄUML, K.-H. (1995). Deutschsprachige Normen für Vertreter von 48 Kategorien [German normative data for representatives of 48 categories]. Sprache \& Kognition, 14, 39-43.

SpItzer, B., \& B̈̈UmL, K.-H. (2007). Retrieval-induced forgetting in item recognition: Evidence for a reduction in general memory strength. Journal of Experimental Psychology: Learning, Memory, \& Cognition, 33, 863-875.

Spitzer, B., Hanslmayr, S., Opitz, B., Mecklinger, A., \& BäUml, K.-H. (2009). Oscillatory correlates of retrieval-induced forgetting in recognition memory. Journal of Cognitive Neuroscience, 21, 976-990.

Tallon-Baudry, C., Bertrand, O., Delpuech, C., \& Permier, J. (1996). Stimulus specificity of phase-locked and non-phase-locked $40 \mathrm{~Hz}$ visual responses in human. Journal of Neuroscience, 16, 42404249.

Veling, H., \& VAN KNIPPEnberG, A. (2004). Remembering can cause inhibition: Retrieval-induced forgetting as cue independent process. Journal of Experimental Psychology: Learning, Memory, \& Cognition, 30, 315-318.

Williams, C. C., \& ZACKs, R. T. (2001). Is retrieval-induced forgetting an inhibitory process? American Journal of Psychology, 114, 329-354.

Wimber, M., Bäuml, K.-H., Bergström, Z., Markopoulos, G., Heinze, H. J., \& Richardson-Klavehn, A. (2008). Neural markers of inhibition in human memory retrieval. Journal of Neuroscience, 28, 13419-13427.

Wimber, M., Rutschmann, R. M., Greenlee, M. W., \& BäUmL, K.-H. (2009). Retrieval from episodic memory: Neural mechanisms of interference resolution. Journal of Cognitive Neuroscience, 21, 538-549.

Yuval-Greenberg, S., Tomer, O., Keren, A. S., Nelken, I., \& Deouell, L. Y. (2008). Transient induced gamma-band response in EEG as a manifestation of miniature saccades. Neuron, 58, 429-441.

\section{NOTES}

1. Concerning the forgetting effects, the $\mathrm{P}-$ items in the competitive condition were recalled significantly less accurately than items in the pooled baseline condition $[57.8 \%$ vs. $66.2 \% ; t(23)=2.87, p<.01]$. $\mathrm{P}-$ items in the noncompetitive condition did not differ from those in the pooled baseline condition $(64.1 \%$ vs. $66.2 \% ; p>.4)$. Concerning the enhancement effects, the $\mathrm{P}+$ items in the competitive condition were recalled better than the items from the pooled baseline $[84.7 \%$ vs. $61.5 \%$; $t(23)=10.01, p<.001] . \mathrm{P}+$ items in the noncompetitive condition were also recalled significantly better than the pooled baseline items $[74.5 \%$ vs. $61.5 \% ; t(23)=5.91, p<.001]$. This additional analysis was conducted to rule out the possibility that the behavioral effects were due to noise in the baseline conditions, which might have amplified the behavioral effects of forgetting and enhancement.

2. No significant difference in the time course of theta phase locking between the frontal and the parietal electrode pools emerged $(p>.05)$. This was ensured by calculating Wilcoxon tests for each time point for theta PLI effects (competitive - noncompetitive) between the frontal and the parietal electrode pools.

3. Correlating theta phase locking with forgetting separately for the frontal and the parietal electrode pools showed that both predicted the amount of subsequent forgetting (frontal, $r=-.52, p<.01$; parietal, $r=-.45, p<.05)$.

\section{SUPPLEMENTAL MATERIALS}

Supplemental figures depicting the effect of retrieval on phase locking as well as the topographies of current-source density transformed ERP effects may be downloaded from http://cabn.psychonomic-journals.org/ content/supplemental.

(Manuscript received October 30, 2009; revision accepted for publication February 24, 2010.) 